\title{
Effects of Various Morphological Traits on Yield Attributes of Cowpea
}

\author{
Anurag J. Greene* \\ BFIT Dehradun, India \\ *Corresponding author
}

\begin{tabular}{|c|c|}
\hline & A B S T R A C T \\
\hline \multicolumn{2}{|l|}{ Keywords } \\
\hline $\begin{array}{l}\text { Path Coefficient, } \\
\text { Direct, Indirect, } \\
\text { Partial Regression, } \\
\text { Genotypic, } \\
\text { Phenotypic }\end{array}$ & \multirow{3}{*}{$\begin{array}{l}\text { Cowpea (Vigna unguiculata) is an important grain legume of the tropics and subtropics, } \\
\text { covering Asia, Africa, and Central and South America, as well as parts of southern Europe } \\
\text { and the United States of America. The present investigation was carried out at Vegetable } \\
\text { Research Farm, Department of Horticulture, SHUATS, Allahabad during the year 2017- } \\
\text { 2018. The experiment was laid out in Randomized Block Design with three replications } \\
\text { having twenty genotypes. The path coefficient analysis is the standardized partial } \\
\text { regression coefficient which splits the correlation coefficient into the measures of direct } \\
\text { and indirect effect of independent variables on the dependent variable. Genotypic and } \\
\text { Phenotypic Path Coefficient Analysis revealed that seed yield }(2.28,1.27) \text { had greatest } \\
\text { positive direct effect on pod yield/plant. }\end{array}$} \\
\hline Article Info & \\
\hline $\begin{array}{l}\text { Accepted: } \\
\text { 20 January } 2019 \\
\text { Available Online: } \\
\text { 10 February } 2019\end{array}$ & \\
\hline
\end{tabular}

\section{Introduction}

Cowpea, Vigna unguiculata (L.) Walp, Leguminosae $(2 \mathrm{n}=2 \mathrm{x}=22)$, is an essential food crop in less-developed countries of the tropics and subtropics, especially in subSaharan Africa, Asia, and Central and South America (Singh et al., 1997). The wild forms are endemic to Africa (Pasquet, 1999; Coulibaly et al., 2002). It is the second most important grain legume crop after groundnut as well as second only to cereals (Blade et al., 1997). As cowpea is native to West Africa where wild and weedy forms exist in many parts of the region ( $\mathrm{Ng}$ and Marechal, 1985), it is one of the most variable species and genetic variability is the basis of genetic enhancement.

\section{Materials and Methods}

The experiment was conducted at Vegetable Research Farm, Department of Horticulture, Naini Agricultural Institute, Sam Higg in bottom University of Agriculture, Technology and Sciences, Allahabad (UP) during 20172018. The experiment comprised of 20 genotypes of cowpea collected from IIVR, Varanasi. Parameters under study were Plant height at first flowering $(\mathrm{cm})$, Plant height at maturity $(\mathrm{cm})$, Number of nodes on the main stem, Days to first flower formation, Days to first pod formation, Days to $50 \%$ flowering, Days to $50 \%$ maturity, Number of pods per peduncle, Number of pods per plant, Number of peduncle per plant, Dry Pod weight $(\mathrm{g})$, 
Pod length $(\mathrm{cm})$, Pod yield (kg/ha), Test weight $(\mathrm{g})$, Seed yield $(\mathrm{kg} / \mathrm{ha})$, Number of seeds per pod, Number of seeds per plant, Seed weight per plant $(\mathrm{g})$, Protein content (\%), TSS content ( ${ }^{\circ}$ brix). Path coefficient analysis suggested by Wright (1921) and illustrated by Dewey and Lu (1959) was carried out separately to know the direct and indirect effects of the important component traits on fruit yield per plant.

Standard path coefficients which are the standardised partial regression coefficient were obtained by solving the following set of ' $\mathrm{P}$ ' simultaneous equations through the use of Doolittle technique as given by Goulden (1959).

\section{Results and Discussion}

\section{Path coefficient analysis}

Correlation coefficient values do not reveal the real association pattern of the independent variables with the dependent one. This approach is more important to comprehend genetic makeup of independent trait when the determining component characters are correlated.

\section{Genotypic path coefficient analysis}

Path coefficients which are worked out from genotypic correlation coefficient are referred to as genotypic path coefficient analysis. It splits the genotypic correlation coefficient into the measures of direct and indirect effect. In other words, it measures the direct and indirect contribution of various independent characters towards a dependent character, say yield in plant breeding experiments.

\section{Phenotypic path coefficient analysis}

Path coefficients which are worked out from phenotypic correlation coefficient are referred to as phenotypic path coefficient analysis. All possible phenotypic correlation coefficients among various characters under study are used for the estimation of phenotypic path. It splits the phenotypic correlation coefficient into the measures of direct and indirect effects.

\section{Seed yield}

\section{Genotypic path coefficient}

At genotypic level, plant height at flowering (0.6761), nodes on main stem (0.2386), days to first pod formation (0.3931), pods per peduncle (0.0132), pods per plant (0.3658), dry pod weight (0.1536), seeds per pod (0.4887), seeds per plant (0.0126), Test weight (0.3608), TSS (0.0576), protein content (0.0392), pod yield (0.4497) had direct positive effect on seed yield per hectare. Similar findings were reported by Kwon and Kwala (2017) for pods per plant, seeds per pod, plant height.

However, the negative direct effect on seed yield per plant was exerted by plant height at maturity (-0.7028), days to first flowering ($0.1165)$, days to $50 \%$ flowering $(-0.2075)$, days to $50 \%$ maturity $(-0.1249)$, peduncles per plant (-0.0843), pod length (-0.0432), seed weight per plant $(-0.0325)$ at genotypic level. Results are represented in table $1 \mathrm{a}$ and $1 \mathrm{~b}$.

\section{Phenotypic path coefficient}

At phenotypic level, plant height at flowering (0.0711), nodes on main stem (0.0652), days to first flowering (0.0132), days to $50 \%$ flowering (0.0953), days to $50 \%$ maturity (0.0149), dry pod weight (0.1946), seeds per pod (0.3176), seeds per plant (0.3493), seed weight per plant(0.0458), Test weight (0.3663), TSS (0.0331), protein content \% (0.0003), pod yield (0.6049) had direct positive effect on seed yield per plant. Results are represented in table $2 \mathrm{a}$ and $2 \mathrm{~b}$. 
Table.1a Genotypic path matrix of seed yield

\begin{tabular}{|c|c|c|c|c|c|c|c|c|c|c|c|}
\hline No. & Character & $\begin{array}{c}\text { Plant } \\
\text { Height At } \\
\text { Flowering } \\
\quad(\mathrm{cm})\end{array}$ & $\begin{array}{c}\text { Plant } \\
\text { Height At } \\
\text { Maturity } \\
\text { (cm) }\end{array}$ & $\begin{array}{c}\text { Nodes } \\
\text { On Main } \\
\text { Stem }\end{array}$ & $\begin{array}{c}\text { Days to } \\
\text { First Pod } \\
\text { Formation }\end{array}$ & $\begin{array}{c}\text { Days to } \\
\text { First } \\
\text { Flowering }\end{array}$ & $\begin{array}{c}\text { Days to } \\
50 \% \\
\text { Flowering }\end{array}$ & $\begin{array}{c}\text { Days to } \\
50 \% \\
\text { Maturity }\end{array}$ & $\begin{array}{c}\text { Peduncles/ } \\
\text { Plant }\end{array}$ & $\begin{array}{c}\text { Pods/ } \\
\text { Peduncle }\end{array}$ & $\begin{array}{l}\text { Pods / } \\
\text { Plant }\end{array}$ \\
\hline 1 & Plant Height at Flowering $(\mathrm{cm})$ & 0.6761 & 0.6745 & 0.0994 & 0.0548 & 0.0404 & 0.0799 & 0.1403 & -0.2770 & 0.0902 & -0.3254 \\
\hline 2 & Plant Height at Maturity $(\mathrm{cm})$ & -0.7012 & -0.7028 & -0.1199 & -0.0642 & -0.0524 & -0.0968 & -0.1460 & 0.2776 & -0.0900 & 0.3097 \\
\hline 3 & Nodes On Main Stem & 0.0351 & 0.0407 & 0.2386 & 0.1151 & 0.1232 & 0.1570 & 0.1315 & -0.0149 & 0.0472 & 0.0705 \\
\hline 4 & Days to First Pod Formation & 0.0318 & 0.0359 & 0.1896 & 0.3931 & 0.3912 & 0.3713 & 0.3271 & -0.0256 & 0.1391 & 0.0901 \\
\hline 5 & Days to First Flowering & -0.0070 & -0.0087 & -0.0602 & -0.1160 & -0.1165 & -0.1113 & -0.0930 & 0.0006 & -0.0439 & -0.0363 \\
\hline 6 & Days to $50 \%$ Flowering & -0.0245 & -0.0286 & -0.1366 & -0.1960 & -0.1982 & -0.2075 & -0.1806 & 0.0216 & -0.0838 & -0.0693 \\
\hline 7 & Days to $50 \%$ Maturity & -0.0259 & -0.0260 & -0.0688 & -0.1040 & -0.0997 & -0.1087 & -0.1249 & 0.0390 & -0.0489 & -0.0029 \\
\hline 8 & Peduncles/ Plant & 0.0345 & 0.0333 & 0.0053 & 0.0055 & 0.0004 & 0.0088 & 0.0263 & -0.0843 & 0.0343 & -0.0427 \\
\hline 9 & Pods/ Peduncle & 0.0018 & 0.0017 & 0.0026 & 0.0047 & 0.0050 & 0.0053 & 0.0051 & -0.0054 & 0.0132 & 0.0010 \\
\hline 10 & Pods / Plant & -0.1761 & -0.1612 & 0.1080 & 0.0838 & 0.1139 & 0.1222 & 0.0084 & 0.1853 & 0.0267 & 0.3658 \\
\hline 11 & Dry Pod Weight (g) & 0.0207 & 0.0250 & 0.0713 & 0.0695 & 0.0764 & 0.0606 & 0.0266 & 0.0480 & 0.0159 & 0.0493 \\
\hline 12 & Pod Length $(\mathrm{cm})$ & -0.0062 & -0.0053 & 0.0021 & -0.0095 & -0.0088 & -0.0096 & -0.0162 & 0.0058 & -0.0213 & 0.0093 \\
\hline 13 & Seeds/ Pod & 0.0303 & 0.0234 & -0.0151 & -0.1494 & -0.1391 & -0.1310 & -0.0621 & -0.0010 & 0.0124 & 0.0081 \\
\hline 14 & Seeds/ Plant & -0.0051 & -0.0048 & 0.0035 & 0.0013 & 0.0024 & 0.0027 & -0.0001 & 0.0061 & 0.0006 & 0.0113 \\
\hline 15 & Seed Weight/ Plant (g) & 0.0104 & 0.0101 & -0.0093 & 0.0064 & 0.0043 & 0.0007 & 0.0075 & -0.0089 & -0.0037 & -0.0213 \\
\hline 16 & Test Weight (g) & 0.1655 & 0.1571 & 0.0052 & -0.0659 & -0.0790 & -0.0570 & -0.0419 & -0.1118 & -0.0379 & -0.1607 \\
\hline 17 & TSS ( ${ }^{0}$ brix $)$ & 0.0116 & 0.0135 & 0.0183 & 0.0052 & 0.0090 & 0.0156 & 0.0093 & 0.0111 & -0.0041 & 0.0193 \\
\hline 18 & Protein Content (\%) & -0.0118 & -0.0110 & 0.0135 & 0.0064 & 0.0086 & 0.0100 & 0.0064 & 0.0118 & -0.0019 & 0.0143 \\
\hline 19 & Pod Yield (kg/ha) & -0.1435 & -0.1250 & 0.2136 & 0.1783 & 0.2059 & 0.1911 & 0.0610 & 0.1779 & 0.0761 & 0.3812 \\
\hline \multirow[t]{2}{*}{20} & Seed Yield (kg/ha) & -0.0835 & -0.0583 & 0.5610 & 0.2192 & 0.2870 & 0.3033 & 0.0849 & 0.2560 & 0.1202 & 0.6711 \\
\hline & Partial R2 & -0.0564 & 0.0410 & 0.1339 & 0.0862 & -0.0335 & -0.0629 & -0.0106 & -0.0216 & 0.0016 & 0.2455 \\
\hline
\end{tabular}

R SQUARE $=1.0003$, RESIDUAL EFFECT $=$ SQRT $(1-1.0003)$ 
Table.1b Genotypic Path Matrix of seed yield (cont.)

\begin{tabular}{|c|c|c|c|c|c|c|c|c|c|c|}
\hline No. & Character & $\begin{array}{c}\text { Dry Pod } \\
\text { Weight } \\
\text { (g) }\end{array}$ & $\begin{array}{l}\text { Pod Length } \\
\text { (cm) }\end{array}$ & Seeds/ Pod & $\begin{array}{l}\text { Seeds/ } \\
\text { Plant }\end{array}$ & $\begin{array}{c}\text { Seed } \\
\text { Weight/ } \\
\text { Plant } \\
\text { (g) }\end{array}$ & $\begin{array}{c}\text { Test } \\
\text { Weight } \\
\text { (g) }\end{array}$ & $\begin{array}{c}\text { TSS } \\
\left({ }^{0} \text { brix }\right)\end{array}$ & $\begin{array}{c}\text { Protein } \\
\text { Content } \\
\quad(\%)\end{array}$ & $\begin{array}{l}\text { Pod Yield } \\
\text { (kg/ha) }\end{array}$ \\
\hline 1 & Plant Height at Flowering $(\mathrm{cm})$ & 0.0911 & 0.0966 & 0.0419 & -0.2751 & -0.2165 & 0.3100 & 0.1365 & -0.2033 & -0.2157 \\
\hline 2 & Plant Height at Maturity $(\mathrm{cm})$ & -0.1142 & -0.0862 & -0.0336 & 0.2665 & 0.2183 & -0.3059 & -0.1642 & 0.1963 & 0.1954 \\
\hline 3 & Nodes On Main Stem & 0.1107 & -0.0114 & -0.0074 & 0.0668 & 0.0682 & 0.0034 & 0.0757 & 0.0822 & 0.1133 \\
\hline 4 & Days to First Pod Formation & 0.1779 & 0.0860 & -0.1202 & 0.0413 & -0.0772 & -0.0718 & 0.0356 & 0.0640 & 0.1559 \\
\hline 5 & Days to First Flowering & -0.0580 & -0.0236 & 0.0332 & -0.0223 & 0.0152 & 0.0255 & -0.0182 & -0.0256 & -0.0534 \\
\hline 6 & Days to $50 \%$ Flowering & -0.0819 & -0.0462 & 0.0556 & -0.0451 & 0.0044 & 0.0328 & -0.0561 & -0.0528 & -0.0882 \\
\hline 7 & Days to $50 \%$ Maturity & -0.0217 & -0.0468 & 0.0159 & 0.0007 & 0.0287 & 0.0145 & -0.0202 & -0.0205 & -0.0169 \\
\hline 8 & Peduncles/ Plant & -0.0264 & 0.0113 & 0.0002 & -0.0412 & -0.0229 & 0.0261 & -0.0163 & -0.0253 & -0.0333 \\
\hline 9 & Pods/ Peduncle & 0.0014 & 0.0065 & 0.0003 & 0.0007 & 0.0015 & -0.0014 & -0.0009 & -0.0006 & 0.0022 \\
\hline 10 & Pods / Plant & 0.1174 & -0.0789 & 0.0060 & 0.3282 & 0.2400 & -0.1628 & 0.1227 & 0.1331 & 0.3100 \\
\hline 11 & Dry Pod Weight (g) & 0.1536 & 0.0183 & 0.0044 & 0.0463 & 0.0050 & -0.0215 & 0.0397 & 0.0160 & 0.1022 \\
\hline 12 & Pod Length (cm) & -0.0051 & -0.0432 & -0.0173 & 0.0005 & 0.0002 & 0.0039 & -0.0053 & 0.0177 & 0.0082 \\
\hline 13 & Seeds/ Pod & 0.0139 & 0.1957 & 0.4887 & 0.2167 & 0.2302 & -0.0163 & -0.0022 & -0.1548 & -0.0009 \\
\hline 14 & Seeds/ Plant & 0.0038 & -0.0002 & 0.0056 & 0.0126 & 0.0099 & -0.0051 & 0.0041 & 0.0029 & 0.0094 \\
\hline 15 & Seed Weight/ Plant (g) & -0.0011 & 0.0001 & -0.0153 & -0.0256 & -0.0325 & 0.0027 & -0.0045 & 0.0020 & -0.0151 \\
\hline 16 & Test Weight (g) & -0.0505 & -0.0323 & -0.0120 & -0.1463 & -0.0294 & 0.3608 & -0.0695 & -0.0563 & -0.1499 \\
\hline 17 & TSS ( ${ }^{0}$ brix $)$ & 0.0149 & 0.0070 & -0.0003 & 0.0189 & 0.0080 & -0.0111 & 0.0576 & 0.0189 & 0.0145 \\
\hline 18 & Protein Content (\%) & 0.0041 & -0.0160 & -0.0124 & 0.0090 & -0.0024 & -0.0061 & 0.0129 & 0.0392 & 0.0141 \\
\hline 19 & Pod Yield (kg/ha) & 0.2993 & -0.0851 & -0.0008 & 0.3354 & 0.2088 & -0.1869 & 0.1133 & 0.1617 & 0.4497 \\
\hline \multirow[t]{2}{*}{20} & Seed Yield (kg/ha) & 0.6292 & -0.0485 & 0.4325 & 0.7879 & 0.6573 & -0.0092 & 0.2406 & 0.1948 & 0.8015 \\
\hline & Partial R² & 0.0967 & 0.0021 & 0.2113 & 0.0099 & -0.0214 & -0.0033 & 0.0139 & 0.0076 & 0.3604 \\
\hline
\end{tabular}

R SQUARE $=1.0003$, RESIDUAL EFFECT =SQRT $(1-\quad 1.0003)$ 
Table.2a Phenotypic path matrix of seed yield

\begin{tabular}{|c|c|c|c|c|c|c|c|c|c|c|c|}
\hline No. & Character & $\begin{array}{c}\text { Plant } \\
\text { Height At } \\
\text { Flowering } \\
\quad(\mathrm{cm})\end{array}$ & $\begin{array}{l}\text { Plant } \\
\text { Height At } \\
\text { Maturity } \\
\quad(\mathrm{cm})\end{array}$ & $\begin{array}{l}\text { Nodes } \\
\text { On } \\
\text { Main } \\
\text { Stem }\end{array}$ & $\begin{array}{l}\text { Days to First } \\
\text { Pod } \\
\text { Formation }\end{array}$ & $\begin{array}{c}\text { Days to } \\
\text { First } \\
\text { Flowering }\end{array}$ & $\begin{array}{c}\text { Days to } \\
50 \% \\
\text { Flowering }\end{array}$ & $\begin{array}{c}\text { Days to } 50 \\
\% \\
\text { Maturity }\end{array}$ & $\begin{array}{l}\text { Peduncles/ } \\
\text { Plant }\end{array}$ & $\begin{array}{c}\text { Pods/ } \\
\text { Peduncle }\end{array}$ & $\begin{array}{l}\text { Pods / } \\
\text { Plant }\end{array}$ \\
\hline $\mathbf{1}$ & Plant Height at Flowering (cm) & 0.0711 & 0.0709 & 0.0100 & 0.0057 & 0.0042 & 0.0084 & 0.0148 & -0.0281 & 0.0086 & -0.0340 \\
\hline 2 & Plant Height at Maturity (cm) & -0.1262 & -0.1266 & -0.0209 & -0.0113 & -0.0093 & -0.0171 & -0.0263 & 0.0479 & -0.0144 & 0.0554 \\
\hline 3 & Nodes On Main Stem & 0.0091 & 0.0108 & 0.0652 & 0.0292 & 0.0324 & 0.0397 & 0.0340 & -0.0021 & 0.0132 & 0.0181 \\
\hline 4 & Days to First Pod Formation & -0.0048 & -0.0053 & -0.0268 & -0.0596 & -0.0583 & -0.0549 & -0.0486 & 0.0039 & -0.0195 & -0.0132 \\
\hline 5 & Days to First Flowering & 0.0008 & 0.0010 & 0.0065 & 0.0129 & 0.0132 & 0.0123 & 0.0104 & 0.0000 & 0.0045 & 0.0040 \\
\hline 6 & Days to $50 \%$ Flowering & 0.0112 & 0.0129 & 0.0582 & 0.0878 & 0.0892 & 0.0953 & 0.0822 & -0.0089 & 0.0348 & 0.0313 \\
\hline 7 & Days to $50 \%$ Maturity & 0.0031 & 0.0031 & 0.0078 & 0.0121 & 0.0118 & 0.0129 & 0.0149 & -0.0045 & 0.0053 & 0.0004 \\
\hline 8 & Peduncles/ Plant & 0.0247 & 0.0237 & 0.0021 & 0.0041 & 0.0001 & 0.0058 & 0.0187 & -0.0625 & 0.0249 & -0.0304 \\
\hline 9 & Pods/ Peduncle & -0.0003 & -0.0003 & -0.0005 & -0.0007 & -0.0008 & -0.0008 & -0.0008 & 0.0009 & -0.0022 & -0.0001 \\
\hline 10 & Pods / Plant & 0.0721 & 0.0660 & -0.0418 & -0.0335 & -0.0461 & -0.0496 & -0.0036 & -0.0734 & -0.0096 & -0.1508 \\
\hline 11 & Dry Pod Weight (g) & 0.0244 & 0.0299 & 0.0812 & 0.0857 & 0.0937 & 0.0738 & 0.0312 & 0.0556 & 0.0165 & 0.0609 \\
\hline 12 & Pod Length $(\mathrm{cm})$ & -0.0134 & -0.0115 & 0.0045 & -0.0196 & -0.0186 & -0.0209 & -0.0351 & 0.0125 & -0.0425 & 0.0187 \\
\hline 13 & Seeds/ Pod & 0.0195 & 0.0148 & -0.0095 & -0.0942 & -0.0880 & -0.0826 & -0.0398 & 0.0005 & 0.0100 & 0.0057 \\
\hline 14 & Seeds/ Plant & -0.1419 & -0.1323 & 0.0938 & 0.0359 & 0.0664 & 0.0748 & -0.0018 & 0.1656 & 0.0166 & 0.3117 \\
\hline 15 & Seed Weight/ Plant (g) & -0.0146 & -0.0142 & 0.0123 & -0.0089 & -0.0060 & -0.0008 & -0.0105 & 0.0121 & 0.0047 & 0.0301 \\
\hline 16 & Test Weight (g) & 0.1678 & 0.1592 & 0.0050 & -0.0654 & -0.0797 & -0.0570 & -0.0424 & -0.1099 & -0.0354 & -0.1621 \\
\hline 17 & TSS ( ${ }^{0}$ brix) & 0.0062 & 0.0073 & 0.0101 & 0.0023 & 0.0047 & 0.0077 & 0.0049 & 0.0067 & -0.0025 & 0.0100 \\
\hline 18 & Protein Content (\%) & -0.0001 & -0.0001 & 0.0001 & 0.0000 & 0.0001 & 0.0001 & 0.0000 & 0.0001 & 0.0000 & 0.0001 \\
\hline 19 & Pod Yield (kg/ha) & -0.1925 & -0.1677 & 0.2736 & 0.2374 & 0.2749 & 0.2535 & 0.0815 & 0.2316 & 0.0933 & 0.5096 \\
\hline \multirow[t]{2}{*}{20} & Seed Yield (kg/ha) & -0.0840 & -0.0583 & 0.5309 & 0.2200 & 0.2837 & 0.3005 & 0.0838 & 0.2481 & 0.1064 & 0.6654 \\
\hline & Partial R ${ }^{2}$ & -0.0060 & 0.0074 & 0.0346 & -0.0131 & 0.0037 & 0.0286 & 0.0012 & -0.0155 & -0.0002 & -0.1003 \\
\hline
\end{tabular}


Table.2b Phenotypic path matrix of seed yield (cont.)

\begin{tabular}{|c|c|c|c|c|c|c|c|c|c|c|}
\hline No. & Character & $\begin{array}{c}\text { Dry Pod } \\
\text { Weight } \\
\text { (g) }\end{array}$ & $\begin{array}{l}\text { Pod Length } \\
(\mathrm{cm})\end{array}$ & $\begin{array}{l}\text { Seeds/ } \\
\text { Pod }\end{array}$ & $\begin{array}{l}\text { Seeds/ } \\
\text { Plant }\end{array}$ & $\begin{array}{c}\text { Seed } \\
\text { Weight/ } \\
\text { Plant } \\
\text { (g) }\end{array}$ & $\begin{array}{c}\text { Test } \\
\text { Weight } \\
\text { (g) }\end{array}$ & $\begin{array}{c}\text { TSS } \\
\left({ }^{0} \text { brix }\right)\end{array}$ & $\begin{array}{c}\text { Protein } \\
\text { Content } \\
(\%)\end{array}$ & $\begin{array}{c}\text { Pod Yield } \\
\text { (kg/ha) }\end{array}$ \\
\hline 1 & Plant Height at Flowering $(\mathrm{cm})$ & 0.0089 & 0.0100 & 0.0044 & -0.0289 & -0.0227 & 0.0326 & 0.0132 & -0.0195 & -0.0226 \\
\hline 2 & Plant Height at Maturity $(\mathrm{cm})$ & -0.0195 & -0.0153 & -0.0059 & 0.0479 & 0.0393 & -0.0550 & -0.0279 & 0.0323 & 0.0351 \\
\hline 3 & Nodes On Main Stem & 0.0272 & -0.0031 & -0.0019 & 0.0175 & 0.0175 & 0.0009 & 0.0198 & 0.0227 & 0.0295 \\
\hline 4 & Days to First Pod Formation & -0.0263 & -0.0123 & 0.0177 & -0.0061 & 0.0116 & 0.0107 & -0.0042 & -0.0095 & -0.0234 \\
\hline 5 & Days to First Flowering & 0.0063 & 0.0026 & -0.0036 & 0.0025 & -0.0017 & -0.0029 & 0.0019 & 0.0027 & 0.0060 \\
\hline 6 & Days to $50 \%$ Flowering & 0.0361 & 0.0209 & -0.0248 & 0.0204 & -0.0017 & -0.0148 & 0.0221 & 0.0221 & 0.0400 \\
\hline 7 & Days to $50 \%$ Maturity & 0.0024 & 0.0055 & -0.0019 & -0.0001 & -0.0034 & -0.0017 & 0.0022 & 0.0022 & 0.0020 \\
\hline 8 & Peduncles/ Plant & -0.0178 & 0.0082 & -0.0001 & -0.0296 & -0.0165 & 0.0187 & -0.0127 & -0.0194 & -0.0239 \\
\hline 9 & Pods/ Peduncle & -0.0002 & -0.0010 & -0.0001 & -0.0001 & -0.0002 & 0.0002 & 0.0002 & 0.0000 & -0.0003 \\
\hline 10 & Pods / Plant & -0.0472 & 0.0296 & -0.0027 & -0.1346 & -0.0990 & 0.0667 & -0.0458 & -0.0486 & -0.1270 \\
\hline 11 & Dry Pod Weight (g) & 0.1946 & 0.0233 & 0.0049 & 0.0566 & 0.0069 & -0.0263 & 0.0435 & 0.0122 & 0.1265 \\
\hline 12 & Pod Length $(\mathrm{cm})$ & -0.0114 & -0.0953 & -0.0375 & 0.0011 & -0.0004 & 0.0084 & -0.0094 & 0.0369 & 0.0177 \\
\hline 13 & Seeds/ Pod & 0.0080 & 0.1251 & 0.3176 & 0.1398 & 0.1492 & -0.0106 & -0.0019 & -0.0902 & -0.0005 \\
\hline 14 & Seeds/ Plant & 0.1016 & -0.0042 & 0.1537 & 0.3493 & 0.2744 & -0.1416 & 0.1075 & 0.0739 & 0.2601 \\
\hline 15 & Seed Weight/ Plant (g) & 0.0016 & 0.0002 & 0.0215 & 0.0360 & 0.0458 & -0.0037 & 0.0057 & -0.0028 & 0.0212 \\
\hline 16 & Test Weight (g) & -0.0496 & -0.0322 & -0.0122 & -0.1485 & -0.0298 & 0.3663 & -0.0661 & -0.0526 & -0.1520 \\
\hline 17 & TSS ( ${ }^{0}$ brix $)$ & 0.0074 & 0.0033 & -0.0002 & 0.0102 & 0.0041 & -0.0060 & 0.0331 & 0.0115 & 0.0077 \\
\hline 18 & Protein Content $(\%)$ & 0.0000 & -0.0001 & -0.0001 & 0.0001 & 0.0000 & 0.0000 & 0.0001 & 0.0003 & 0.0001 \\
\hline 19 & Pod Yield (kg/ha) & 0.3931 & -0.1121 & -0.0009 & 0.4505 & 0.2800 & -0.2510 & 0.1407 & 0.1972 & 0.6049 \\
\hline \multirow[t]{2}{*}{20} & Seed Yield (kg/ha) & 0.6153 & -0.0469 & 0.4279 & 0.7841 & 0.6534 & -0.0092 & 0.2222 & 0.1712 & 0.8009 \\
\hline & Partial R² & 0.1197 & 0.0045 & 0.1359 & 0.2739 & 0.0300 & -0.0034 & 0.0074 & 0.0000 & 0.4845 \\
\hline
\end{tabular}

R SQUARE $=1.0003$, RESIDUAL EFFECT $=$ SQRT $(1-1.000)$ 
Table.3 Genotypic path matrix of pod yield

\begin{tabular}{|c|c|c|c|c|c|c|c|c|c|c|c|}
\hline No. & Character & $\begin{array}{l}\text { Plant } \\
\text { Height At } \\
\text { Flowering } \\
\quad \text { (cm) }\end{array}$ & $\begin{array}{l}\text { Plant } \\
\text { Height At } \\
\text { Maturity } \\
\quad(\mathrm{cm})\end{array}$ & $\begin{array}{l}\text { Nodes } \\
\text { On Main } \\
\text { Stem }\end{array}$ & $\begin{array}{c}\text { Days to } \\
\text { First Pod } \\
\text { Formation }\end{array}$ & $\begin{array}{c}\text { Days to } \\
\text { First } \\
\text { Flowering }\end{array}$ & $\begin{array}{c}\text { Days to } \\
50 \% \\
\text { Flowering }\end{array}$ & $\begin{array}{c}\text { Days to } \\
50 \% \\
\text { Maturity }\end{array}$ & $\begin{array}{l}\text { Peduncles/ } \\
\text { Plant }\end{array}$ & $\begin{array}{c}\text { Pods/ } \\
\text { Peduncle }\end{array}$ & $\begin{array}{l}\text { Pods / } \\
\text { Plant }\end{array}$ \\
\hline 1 & Plant Height at Flowering $(\mathrm{cm})$ & -1.5359 & -1.5324 & -0.2259 & -0.1244 & -0.0917 & -0.1816 & -0.3188 & 0.6294 & -0.2049 & 0.7393 \\
\hline 2 & Plant Height at Maturity $(\mathrm{cm})$ & 1.6023 & 1.6060 & 0.2740 & 0.1467 & 0.1197 & 0.2211 & 0.3336 & -0.6342 & 0.2057 & -0.7078 \\
\hline 3 & Nodes On Main Stem & -0.0790 & -0.0916 & -0.5368 & -0.2589 & -0.2772 & -0.3533 & -0.2958 & 0.0335 & -0.1062 & -0.1585 \\
\hline 4 & Days to First Pod Formation & -0.0721 & -0.0814 & -0.4296 & -0.8908 & -0.8864 & -0.8413 & -0.7411 & 0.0580 & -0.3153 & -0.2041 \\
\hline 5 & Days to First Flowering & 0.0148 & 0.0184 & 0.1277 & 0.2462 & 0.2474 & 0.2363 & 0.1973 & -0.0013 & 0.0932 & 0.0770 \\
\hline 6 & Days to $50 \%$ Flowering & 0.0564 & 0.0657 & 0.3140 & 0.4506 & 0.4557 & 0.4771 & 0.4153 & -0.0498 & 0.1927 & 0.1594 \\
\hline 7 & Days to $50 \%$ Maturity & 0.0562 & 0.0562 & 0.1492 & 0.2252 & 0.2159 & 0.2356 & 0.2707 & -0.0844 & 0.1059 & 0.0062 \\
\hline 8 & Peduncles/ Plant & -0.0758 & -0.0730 & -0.0116 & -0.0120 & -0.0009 & -0.0193 & -0.0577 & 0.1849 & -0.0753 & 0.0937 \\
\hline 9 & Pods/ Peduncle & -0.0047 & -0.0045 & -0.0070 & -0.0126 & -0.0134 & -0.0143 & -0.0139 & 0.0145 & -0.0355 & -0.0026 \\
\hline 10 & Pods / Plant & 0.4466 & 0.4089 & -0.2740 & -0.2126 & -0.2889 & -0.3100 & -0.0213 & -0.4699 & -0.0678 & -0.9278 \\
\hline 11 & Dry Pod Weight (g) & -0.0496 & -0.0599 & -0.1710 & -0.1668 & -0.1833 & -0.1455 & -0.0639 & -0.1153 & -0.0381 & -0.1183 \\
\hline 12 & Pod Length $(\mathrm{cm})$ & 0.0167 & 0.0143 & -0.0056 & 0.0255 & 0.0237 & 0.0260 & 0.0437 & -0.0157 & 0.0574 & -0.0252 \\
\hline 13 & Seeds/ Pod & -0.0726 & -0.0559 & 0.0361 & 0.3579 & 0.3332 & 0.3138 & 0.1487 & 0.0023 & -0.0297 & -0.0193 \\
\hline 14 & Seeds/ Plant & -0.0348 & -0.0324 & 0.0239 & 0.0090 & 0.0164 & 0.0186 & -0.0005 & 0.0418 & 0.0044 & 0.0767 \\
\hline 15 & Seed Weight/ Plant (g) & -0.0178 & -0.0173 & 0.0159 & -0.0109 & -0.0073 & -0.0012 & -0.0128 & 0.0151 & 0.0064 & 0.0365 \\
\hline 16 & Test Weight (g) & -0.3784 & -0.3592 & -0.0119 & 0.1507 & 0.1806 & 0.1304 & 0.0957 & 0.2557 & 0.0866 & 0.3674 \\
\hline 17 & TSS ( ${ }^{0}$ brix $)$ & -0.0282 & -0.0327 & -0.0444 & -0.0127 & -0.0218 & -0.0378 & -0.0226 & -0.0270 & 0.0101 & -0.0469 \\
\hline 18 & Protein Content (\%) & 0.0280 & 0.0260 & -0.0320 & -0.0151 & -0.0204 & -0.0236 & -0.0153 & -0.0279 & 0.0045 & -0.0338 \\
\hline 19 & Seed Yield (kg/ha) & -0.1910 & -0.1334 & 1.2837 & 0.5015 & 0.6567 & 0.6940 & 0.1942 & 0.5859 & 0.2751 & 1.5357 \\
\hline \multirow[t]{2}{*}{20} & Pod Yield (kg/ha) & -0.3191 & -0.2780 & 0.4749 & 0.3965 & 0.4579 & 0.4250 & 0.1356 & 0.3957 & 0.1692 & 0.8476 \\
\hline & Partial R ${ }^{2}$ & 0.4901 & -0.4465 & -0.2549 & -0.3532 & 0.1133 & 0.2028 & 0.0367 & 0.0732 & -0.0060 & -0.7864 \\
\hline
\end{tabular}

R SQUARE $=1.0003$, RESIDUAL EFFECT $=$ SQRT $(1-1.0003)$ 
Table.4 Genotypic path matrix of pod yield (cont.)

\begin{tabular}{|c|c|c|c|c|c|c|c|c|c|c|}
\hline No. & Character & $\begin{array}{c}\text { Dry Pod } \\
\text { Weight (g) }\end{array}$ & $\begin{array}{c}\text { Pod Length } \\
\text { cm }\end{array}$ & Seeds/ Pod & $\begin{array}{l}\text { Seeds/ } \\
\text { Plant }\end{array}$ & $\begin{array}{c}\text { Seed } \\
\text { Weight/ } \\
\text { Plant (g) }\end{array}$ & $\begin{array}{c}\text { Test Weight } \\
\text { (g) }\end{array}$ & $\begin{array}{c}\text { TSS } \\
\left({ }^{0} \text { brix }\right)\end{array}$ & $\begin{array}{c}\text { Protein } \\
\text { Content } \\
(\%)\end{array}$ & $\begin{array}{c}\text { Seed Yield } \\
\text { (kg/ha) }\end{array}$ \\
\hline 1 & Plant Height at Flowering $(\mathrm{cm})$ & -0.2069 & -0.2194 & -0.0953 & 0.6249 & 0.4918 & -0.7043 & -0.3102 & 0.4618 & 0.1282 \\
\hline 2 & Plant Height at Maturity (cm) & 0.2609 & 0.1970 & 0.0768 & -0.6089 & -0.4988 & 0.6990 & 0.3753 & -0.4485 & -0.0936 \\
\hline 3 & Nodes On Main Stem & -0.2490 & 0.0256 & 0.0166 & -0.1502 & -0.1535 & -0.0077 & -0.1703 & -0.1850 & -0.3012 \\
\hline 4 & Days to First Pod Formation & -0.4032 & -0.1948 & 0.2724 & -0.0935 & 0.1749 & 0.1627 & -0.0807 & -0.1450 & -0.1952 \\
\hline 5 & Days to First Flowering & 0.1230 & 0.0502 & -0.0704 & 0.0474 & -0.0323 & -0.0541 & 0.0387 & 0.0543 & 0.0710 \\
\hline 6 & Days to $50 \%$ Flowering & 0.1883 & 0.1062 & -0.1279 & 0.1037 & -0.0101 & -0.0754 & 0.1291 & 0.1213 & 0.1447 \\
\hline 7 & Days to $50 \%$ Maturity & 0.0469 & 0.1013 & -0.0344 & -0.0014 & -0.0622 & -0.0314 & 0.0438 & 0.0444 & 0.0230 \\
\hline 8 & Peduncles/ Plant & 0.0578 & -0.0249 & -0.0004 & 0.0905 & 0.0504 & -0.0573 & 0.0357 & 0.0555 & 0.0473 \\
\hline 9 & Pods/ Peduncle & -0.0037 & -0.0175 & -0.0009 & -0.0018 & -0.0041 & 0.0037 & 0.0026 & 0.0017 & -0.0043 \\
\hline 10 & Pods / Plant & -0.2978 & 0.2002 & -0.0153 & -0.8325 & -0.6087 & 0.4131 & -0.3111 & -0.3376 & -0.6227 \\
\hline 11 & Dry Pod Weight (g) & -0.3686 & -0.0438 & -0.0104 & -0.1110 & -0.0120 & 0.0516 & -0.0953 & -0.0385 & -0.2319 \\
\hline 12 & Pod Length $(\mathrm{cm})$ & 0.0139 & 0.1167 & 0.0467 & -0.0014 & -0.0005 & -0.0104 & 0.0142 & -0.0476 & -0.0057 \\
\hline 13 & Seeds/ Pod & -0.0332 & -0.4686 & -1.1703 & -0.5190 & -0.5512 & 0.0389 & 0.0052 & 0.3707 & -0.5061 \\
\hline 14 & Seeds/ Plant & 0.0257 & -0.0011 & 0.0379 & 0.0855 & 0.0673 & -0.0346 & 0.0281 & 0.0196 & 0.0673 \\
\hline 15 & Seed Weight/ Plant (g) & 0.0018 & -0.0002 & 0.0262 & 0.0438 & 0.0556 & -0.0045 & 0.0077 & -0.0034 & 0.0365 \\
\hline 16 & Test Weight (g) & 0.1156 & 0.0738 & 0.0274 & 0.3345 & 0.0673 & -0.8251 & 0.1590 & 0.1287 & 0.0076 \\
\hline 17 & TSS ( ${ }^{0}$ brix $)$ & -0.0361 & -0.0170 & 0.0006 & -0.0460 & -0.0193 & 0.0269 & -0.1398 & -0.0458 & -0.0336 \\
\hline 18 & Protein Content $(\%)$ & -0.0097 & 0.0380 & 0.0295 & -0.0214 & 0.0057 & 0.0145 & -0.0305 & -0.0930 & -0.0181 \\
\hline 19 & Seed Yield (kg/ha) & 1.4397 & -0.1109 & 0.9896 & 1.8028 & 1.5041 & -0.0210 & 0.5505 & 0.4458 & 2.2881 \\
\hline \multirow[t]{2}{*}{20} & Pod Yield (kg/ha) & 0.6655 & -0.1893 & -0.0018 & 0.7459 & 0.4643 & -0.4155 & 0.2519 & 0.3596 & 0.8015 \\
\hline & Partial R ${ }^{2}$ & -0.2453 & -0.0221 & 0.0021 & 0.0637 & 0.0258 & 0.3429 & -0.0352 & -0.0334 & 1.8338 \\
\hline
\end{tabular}

R SQUARE $=1.0003$, RESIDUAL EFFECT $=$ SQRT $(1-1.0003)$ 
Table.5 Phenotypic path matrix of pod yield

\begin{tabular}{|c|c|c|c|c|c|c|c|c|c|c|c|}
\hline No & Character & $\begin{array}{c}\text { Plant } \\
\text { Height At } \\
\text { Flowering } \\
\text { (cm) }\end{array}$ & $\begin{array}{l}\text { Plant } \\
\text { Height At } \\
\text { Maturity } \\
\quad(\mathrm{cm})\end{array}$ & $\begin{array}{l}\text { Nodes } \\
\text { On } \\
\text { Main } \\
\text { Stem }\end{array}$ & $\begin{array}{c}\text { Days to } \\
\text { First Pod } \\
\text { Formation }\end{array}$ & $\begin{array}{c}\text { Days to } \\
\text { First } \\
\text { Flowering }\end{array}$ & $\begin{array}{c}\text { Days to } \\
50 \% \\
\text { Flowering }\end{array}$ & $\begin{array}{c}\text { Days to } \\
50 \% \\
\text { Maturity }\end{array}$ & $\begin{array}{l}\text { Peduncles/ } \\
\text { Plant }\end{array}$ & $\begin{array}{c}\text { Pods/ } \\
\text { Peduncle }\end{array}$ & $\begin{array}{l}\text { Pods / } \\
\text { Plant }\end{array}$ \\
\hline 1 & Plant Height at Flowering $(\mathrm{cm})$ & -0.3892 & -0.3881 & -0.0545 & -0.0312 & -0.0227 & -0.0457 & -0.0808 & 0.1537 & -0.0473 & 0.1860 \\
\hline 2 & Plant Height at Maturity $(\mathrm{cm})$ & 0.4478 & 0.4490 & 0.0743 & 0.0402 & 0.0331 & 0.0608 & 0.0931 & -0.1701 & 0.0510 & -0.1966 \\
\hline 3 & Nodes On Main Stem & -0.0126 & -0.0149 & -0.0902 & -0.0405 & -0.0448 & -0.0550 & -0.0471 & 0.0030 & -0.0183 & -0.0250 \\
\hline 4 & Days to First Pod Formation & 0.0135 & 0.0151 & 0.0758 & 0.1689 & 0.1652 & 0.1555 & 0.1376 & -0.0110 & 0.0554 & 0.0375 \\
\hline 5 & Days to First Flowering & -0.0045 & -0.0057 & -0.0385 & -0.0758 & -0.0775 & -0.0725 & -0.0613 & 0.0002 & -0.0264 & -0.0237 \\
\hline 6 & Days to $50 \%$ Flowering & -0.0233 & -0.0269 & -0.1212 & -0.1828 & -0.1859 & -0.1986 & -0.1713 & 0.0185 & -0.0725 & -0.0653 \\
\hline 7 & Days to $50 \%$ Maturity & 0.0099 & 0.0099 & 0.0250 & 0.0389 & 0.0378 & 0.0412 & 0.0478 & -0.0143 & 0.0169 & 0.0011 \\
\hline 8 & Peduncles/ Plant & -0.0285 & -0.0273 & -0.0024 & -0.0047 & -0.0002 & -0.0067 & -0.0216 & 0.0722 & -0.0288 & 0.0351 \\
\hline 9 & Pods/ Peduncle & 0.0041 & 0.0039 & 0.0069 & 0.0112 & 0.0116 & 0.0124 & 0.0121 & -0.0136 & 0.0340 & 0.0022 \\
\hline 10 & Pods / Plant & -0.1388 & -0.1272 & 0.0805 & 0.0645 & 0.0888 & 0.0955 & 0.0070 & 0.1415 & 0.0185 & 0.2906 \\
\hline 11 & Dry Pod Weight (g) & -0.0163 & -0.0200 & -0.0542 & -0.0573 & -0.0626 & -0.0493 & -0.0209 & -0.0372 & -0.0111 & -0.0407 \\
\hline 12 & Pod Length $(\mathrm{cm})$ & 0.0098 & 0.0084 & -0.0033 & 0.0143 & 0.0136 & 0.0152 & 0.0257 & -0.0091 & 0.0310 & -0.0137 \\
\hline 13 & Seeds/ Pod & -0.0254 & -0.0192 & 0.0123 & 0.1224 & 0.1144 & 0.1074 & 0.0518 & -0.0006 & -0.0131 & -0.0074 \\
\hline 14 & Seeds/ Plant & 0.1739 & 0.1621 & -0.1149 & -0.0440 & -0.0813 & -0.0916 & 0.0023 & -0.2029 & -0.0203 & -0.3818 \\
\hline 15 & Seed Weight/ Plant (g) & 0.0041 & 0.0040 & -0.0035 & 0.0025 & 0.0017 & 0.0002 & 0.0029 & -0.0034 & -0.0013 & -0.0085 \\
\hline 16 & Test Weight (g) & -0.2219 & -0.2106 & -0.0066 & 0.0865 & 0.1054 & 0.0754 & 0.0560 & 0.1453 & 0.0468 & 0.2143 \\
\hline 17 & TSS $\left({ }^{0}\right.$ brix $)$ & -0.0096 & -0.0114 & -0.0157 & -0.0036 & -0.0074 & -0.0120 & -0.0077 & -0.0105 & 0.0039 & -0.0157 \\
\hline 18 & Protein Content $(\%)$ & -0.0040 & -0.0037 & 0.0050 & 0.0023 & 0.0030 & 0.0033 & 0.0022 & 0.0045 & -0.0002 & 0.0046 \\
\hline 19 & Seed Yield (kg/ha) & -0.1072 & -0.0745 & 0.6776 & 0.2808 & 0.3621 & 0.3835 & 0.1070 & 0.3167 & 0.1358 & 0.8493 \\
\hline \multirow[t]{2}{*}{20} & Pod Yield (kg/ha) & -0.3182 & -0.2772 & 0.4522 & 0.3925 & 0.4544 & 0.4191 & 0.1348 & 0.3828 & 0.1542 & 0.8424 \\
\hline & Partial R² & 0.1239 & -0.1245 & -0.0408 & 0.0663 & -0.0352 & -0.0832 & 0.0064 & 0.0276 & 0.0053 & 0.2448 \\
\hline
\end{tabular}

R SQUARE $=1.0003$, RESIDUAL EFFECT $=$ SQRT $(1-1.0003)$ 
Table.6 Phenotypic path matrix of pod yield (cont.)

\begin{tabular}{|c|c|c|c|c|c|c|c|c|c|c|}
\hline No & Character & $\begin{array}{c}\text { Dry Pod } \\
\text { Weight } \\
\text { (g) }\end{array}$ & $\begin{array}{l}\text { Pod Length } \\
\quad(\mathrm{cm})\end{array}$ & Seeds/ Pod & $\begin{array}{l}\text { Seeds/ } \\
\text { Plant }\end{array}$ & $\begin{array}{c}\text { Seed } \\
\text { Weight/ } \\
\text { Plant } \\
\text { (g) }\end{array}$ & $\begin{array}{c}\text { Test Weight } \\
\text { (g) }\end{array}$ & $\begin{array}{c}\text { TSS } \\
\left({ }^{0} \text { brix }\right)\end{array}$ & $\begin{array}{c}\text { Protein } \\
\text { Content } \\
\quad(\%)\end{array}$ & $\begin{array}{l}\text { Seed Yield } \\
\text { (kg/ha) }\end{array}$ \\
\hline 1 & Plant Height at Flowering $(\mathrm{cm})$ & -0.0489 & -0.0547 & -0.0239 & 0.1582 & 0.1243 & -0.1783 & -0.0724 & 0.1070 & 0.0327 \\
\hline 2 & Plant Height at Maturity $(\mathrm{cm})$ & 0.0691 & 0.0543 & 0.0209 & -0.1701 & -0.1393 & 0.1952 & 0.0990 & -0.1144 & -0.0262 \\
\hline 3 & Nodes On Main Stem & -0.0376 & 0.0043 & 0.0027 & -0.0242 & -0.0242 & -0.0012 & -0.0275 & -0.0314 & -0.0479 \\
\hline 4 & Days to First Pod Formation & 0.0744 & 0.0347 & -0.0501 & 0.0174 & -0.0327 & -0.0302 & 0.0119 & 0.0269 & 0.0371 \\
\hline 5 & Days to First Flowering & -0.0373 & -0.0151 & 0.0215 & -0.0147 & 0.0101 & 0.0169 & -0.0110 & -0.0159 & -0.0220 \\
\hline 6 & Days to $50 \%$ Flowering & -0.0753 & -0.0435 & 0.0516 & -0.0425 & 0.0036 & 0.0309 & -0.0461 & -0.0460 & -0.0597 \\
\hline 7 & Days to $50 \%$ Maturity & 0.0077 & 0.0176 & -0.0060 & -0.0003 & -0.0109 & -0.0055 & 0.0071 & 0.0072 & 0.0040 \\
\hline 8 & Peduncles/ Plant & 0.0206 & -0.0095 & 0.0001 & 0.0342 & 0.0190 & -0.0217 & 0.0147 & 0.0224 & 0.0179 \\
\hline 9 & Pods/ Peduncle & 0.0029 & 0.0152 & 0.0011 & 0.0016 & 0.0035 & -0.0033 & -0.0026 & -0.0005 & 0.0036 \\
\hline 10 & Pods / Plant & 0.0909 & -0.0571 & 0.0052 & 0.2593 & 0.1908 & -0.1286 & 0.0882 & 0.0937 & 0.1933 \\
\hline 11 & Dry Pod Weight (g) & -0.1300 & -0.0156 & -0.0033 & -0.0378 & -0.0046 & 0.0176 & -0.0291 & -0.0081 & -0.0800 \\
\hline 12 & Pod Length (cm) & 0.0084 & 0.0696 & 0.0274 & -0.0008 & 0.0003 & -0.0061 & 0.0069 & -0.0270 & -0.0033 \\
\hline 13 & Seeds/ Pod & -0.0103 & -0.1627 & -0.4129 & -0.1817 & -0.1940 & 0.0138 & 0.0024 & 0.1173 & -0.1767 \\
\hline 14 & Seeds/ Plant & -0.1245 & 0.0051 & -0.1883 & -0.4279 & -0.3361 & 0.1734 & -0.1317 & -0.0905 & -0.3355 \\
\hline 15 & Seed Weight/ Plant (g) & -0.0005 & -0.0001 & -0.0060 & -0.0101 & -0.0129 & 0.0010 & -0.0016 & 0.0008 & -0.0084 \\
\hline 16 & Test Weight (g) & 0.0655 & 0.0425 & 0.0162 & 0.1963 & 0.0394 & -0.4843 & 0.0874 & 0.0696 & 0.0045 \\
\hline 17 & TSS ( ${ }^{0}$ brix $)$ & -0.0116 & -0.0051 & 0.0003 & -0.0159 & -0.0065 & 0.0093 & -0.0517 & -0.0180 & -0.0115 \\
\hline 18 & Protein Content $(\%)$ & 0.0009 & -0.0056 & -0.0041 & 0.0030 & -0.0009 & -0.0021 & 0.0050 & 0.0144 & 0.0025 \\
\hline 19 & Seed Yield (kg/ha) & 0.7853 & -0.0598 & 0.5461 & 1.0008 & 0.8340 & -0.0118 & 0.2836 & 0.2186 & 1.2764 \\
\hline \multirow[t]{2}{*}{20} & Pod Yield (kg/ha) & 0.6498 & -0.1853 & -0.0015 & 0.7447 & 0.4629 & -0.4149 & 0.2326 & 0.3260 & 0.8009 \\
\hline & Partial R² & -0.0845 & -0.0129 & 0.0006 & -0.3187 & -0.0060 & 0.2009 & -0.0120 & 0.0047 & 1.0223 \\
\hline
\end{tabular}

$\mathrm{R}$ SQUARE $=1.0003$, RESIDUAL EFFECT $=\mathrm{SQRT}(1-1.0003)$ 
However, the negative direct effect on seed yield per plant was exerted by plant height at maturity (-0.1266), days to first pod formation $(-0.0596)$, peduncles per plant $(-0.0625)$, pods per peduncle $(-0.0022)$, pod length $(-0.0953)$ at phenotypic level.

\section{Pod yield}

\section{Genotypic path coefficient}

At genotypic level, plant height at maturity (1.6060), days to first flowering (0.2474), days to $50 \%$ flowering $(0.4771)$, days to 50 $\%$ maturity (0.2707), peduncles per plant (0.1849), pods per peduncle $(0.1692)$, pod length (0.1167), seeds per plant (0.0855), seed weight per plant (0.0556), seed yield (2.2881) had positive direct effect on pod yield per plant.

The present findings are in conformity with Patel et al., (2016) for days to $50 \%$ flowering, pod length and seeds per plant.

However the negative direct effect on pod yield per plant was exerted by plant height at flowering (-1.5359), nodes on main stem ($0.5368)$, days to first pod formation (-0.8908), pods per peduncle $(-0.0355)$, pods per plant ($0.9278)$, dry pod weight $(-0.3686)$, seeds per pod $(-1.1703)$, test weight $(-0.8251)$, $\operatorname{TSS}^{0}(-$ $0.1398)$, protein content \% (-0.0930) at genotypic level. Results are represented in table 3 and 4.

\section{Phenotypic path coefficient}

At phenotypic level, plant height at maturity (0.4490), days to first pod formation (0.1689), days to $50 \%$ maturity $(0.0478)$, peduncles per plant (0.0722), pods per peduncle (0.0340), pods per plant (0.2906), pod length (0.0696), protein content \% (0.0144), seed yield (1.2764) had positive direct effect on pod yield per plant (Table 5 and 6).
It is concluded as at genotypic and phenotypic level, Nodes on main stem, Days to first Pod Formation, Days to First Flowering, Days to $50 \%$ Flowering, Days to $50 \%$ Maturity, Peduncles per Plant, Pods per Peduncle, Pods per Plant, Dry Pod Weight, Seeds per Plant, Seed Weight per Plant, TSS, Protein Content, Seed Yield had direct positive effect on Pod Yield per Plant. The study reveals significant direct and indirect effects of various morphological traits on the total fruit yield of the crop and helps in estimating those helpful traits which should be considered while undertaking any breeding improvement program in the related crop.

\section{References}

Blade, S.F., Shetty, S.V.R., Terao, T. and Singh, BB. (1997). Recent developments in cowpea cropping systems research, In: Singh BB, Mohan Raj DR, Dashiell KE, Jackai LEN. eds. Advances in Cowpea Research 1997; 114-128, IITA, Ibadan.

Coulibaly, S., Pasquet, R.S., Papa, R. and Gepts, P. (2002). AFLP analysis of the phenetic organization and genetic diversity of Vigna unguiculata L. Walp. Theoretical and Applied Genetics., 104: 358-366.

Dewey, D. R. and Lu, K. H. (1959). A correlation and path analysis of the components of crested wheat grass seed production. Agronomy Journal., 51: 515-518.

Goulden, G. (1959). Method of Statistical Analysis, Asia Publishing House, Calcutta. NHB (2011). Indian Horticulture Database. 178-185.

Kwon-Ndung, E.H. and Kwala, T. D. (2017). Genetic variability, correlation and path coefficient analysis in seed yield and related traits of cowpea germplasm accessions on high altitude 
area of Jos Plateau. The International Journal of Innovative Approaches in Agricultural Research Volume 1 Issue 1: $15-19$.

Ng.; NQ. and Marechal, R. (1985). Cowpea taxonomy, origin and germplasm In: Singh SR, Rachie KO eds. Cowpea Research, Production and Utilization 1985; Chichester, John Wiley and Sons Ltd., 11-21.

Pasquet, R.S. (1999). Genetic relationships among subspecies of Vigna unguiculata (L.) Walp. based on allozyme variation. Theoretical and Applied Genetics 98: 1104-1119.

Patel, U.V., Parmar, V.K., Patel, P.B. and
Malviya, A.V. (2016). Correlation and Path Analysis Study In Cowpea (Vigna unguiculata (L.) Walp.). International Journal of Science, Environment and Technology., Vol. 5, No 6, 2016, 3897 - 3904.

Singh, BB., Mohan Raj, DR., Dashiell, KE. and Jackai, LEN. (1997). Advances in Cowpea Research. Co publication of IITA and JIRCAS. IITA, Ibadan, Nigeria.

Wright, S. (1921). Correlation and causation. Journal of Agriculture Research., 20: 557-587.

\section{How to cite this article:}

Anurag J. Greene 2019. Effects of Various Morphological Traits on Yield Attributes of Cowpea. Int.J.Curr.Microbiol.App.Sci. 8(02): 2836-2847.

doi: https://doi.org/10.20546/ijcmas.2019.802.333 